\title{
The Epidemiology of Acne Vulgaris among Adolescents and Young Adults in Jordan University Hospital
}

\author{
Ruba M. Jaber*, Basma M. Alnshash, Suzan N. Mousa, Hanan S. Fayoumi, Lana M. Al-Qaderi, \\ Asma M. Zant
}

School of Medicine, University of Jordan, Amman, Jordan

Email: ^r.jaber@ju.edu.jo,dr.jaber_ruba@ymail.com

How to cite this paper: Jaber, R.M., Alnshash, B.M., Mousa, S.N., Fayoumi, H.S., Al-Qaderi, L.M. and Zant, A.M. (2020) The Epidemiology of Acne Vulgaris among Adolescents and Young Adults in Jordan University Hospital. Open Journal of Nursing, 10, 353-366.

https://doi.org/10.4236/ojn.2020.104024

Received: February 27, 2020

Accepted: April 6, 2020

Published: April 9, 2020

Copyright (C 2020 by author(s) and Scientific Research Publishing Inc. This work is licensed under the Creative Commons Attribution International License (CC BY 4.0).

http://creativecommons.org/licenses/by/4.0/

\section{(c) (i) Open Access}

\begin{abstract}
Background: Acne vulgaris is a widely prevalent distressing problem among young adults and adolescents. Almost one-third of young people consider acne to be a major problem/burden. Less than half of acne diagnoses are made by a physician, and acne is often self-managed. Objective: We aim to find the prevalence of acne among different age groups, treatment seeking behaviors and sources of knowledge about acne treatment. We also aim to determine impact of acne vulgaris on quality of life and psychology of patients. Design: A cross-sectional study. Setting: Jordan university hospital, Amman, Jordan. Subjects: Patients aged 15 - 35 years old who attended the family medicine clinic in Jordan University Hospital. Intervention: A self-administered questionnaire, consisted of three major parts, distributed during the period between August 2019 and October 2019. Results: Upon analyzing the data, 153 out of the 300 participants were found to have suffered or were still suffering from acne with a prevalence of $45 \%$. The mean age at which the acne started was 16.20 . There was a significant correlation between severity of acne and impact on life. More than half of the affected participants sought doctor help to treat their acne; patients depended on many sources of information to manage their acne. The majority of them reported the dermatologist as their source of information. Patients tended to use different methods of treatment to manage their acne. The cleanser was the most commonly used methods of treatment. Conclusion: Our article demonstrates that acne is a prevalent distressing problem among adolescents; it affects patients' quality of life. Different modalities of treatment and sources of information were sought by patients. As family doctors, we emphasize the need for accessible, accurate, community-based education on the modalities
\end{abstract}


of treatment of acne, and the importance of prompt medical attention toward its effect on quality of life.

\section{Keywords}

Acne Vulgaris, Prevalence, Quality of Life, Treatment, Adolescents, Cyst, Whiteheads, Blackheads, Nodules

\section{Introduction}

Acne vulgaris is a widely prevalent distressing problem among young adults and adolescents, it causes a significant impact on quality of life, social and psychological functioning. It affects over $80 \%$ of teenagers, and persists beyond the age of 25 years in $3 \%$ of men and $12 \%$ of women. It is a common and chronic dermatological disorder of pilosebaceous follicles. Typical lesions of acne include comedones, inflammatory papules, and pustules. Nodules and cysts occur in more severe acne and can cause scarring and psychological distress [1].

Many factors were reported to have affected the prevalence of acne such as race, age and gender. Although it was more prevalent among men at 18; beyond the age of 23 clinical acne was more prevalent among women as the prevalence in men gradually declined [2].

Almost one-third of young people considered acne to be a major problem/burden. Less than half of acne diagnoses are made by a physician, and acne is often self-managed using OTC treatments [3]. Affected individuals tend to deal with their acne in many different ways. Patients used traditional medicine, visited beauty clinics, drank more water, and used over-the-counter topical agents more frequently than they sought doctors' help during the worsening period [4].

Since the majority of patients are getting information from non-physician sources [5], there might be a need to evaluate the resources they are using to make sure they are receiving appropriate, helpful information.

In this study, we aim to find the prevalence of acne among different age groups, treatment seeking behaviors and sources of knowledge about acne treatment. We also aim to determine the impact of acne vulgaris on quality of life and psychology of patients.

\section{Methods}

\subsection{Design and Setting}

This is a cross-sectional study that was conducted at Jordan University Hospital (JUH), which is considered to be the biggest University Hospital in the Capital of Jordan, Amman. Patients aged 15 - 35 years old who attended the family medicine clinic in the period between August 2019 and October 2019 were asked to fill up a self-administered questionnaire

A pilot study was first conducted to test the validity of the questionnaire in 
which a total of fifty questionnaires were distributed on patients and they were asked about their feedback and if they faced any difficulty while filling it up. The mentioned questionnaires were not included in the analysis process.

The questionnaire consisted of three major parts, the first one asked about socio-demographic factors, including age, gender, and marital status, level of education, income and known medical illnesses. The 2 nd part was started with a direct question about having acne in the current period or in the past. Those who answered by yes completed the third part of the questionnaire.

The third part asked about site and type of acne, severity of acne from the patient point of view, the impact of acne on patients' self-esteem and quality of life, the resources patient used to get knowledge, modalities of treatment the patient had used or still using and the amount of money they used to spend on treatment.

Ethical approvals were obtained from all relevant ethical bodies at the University of Jordan and University of Jordan hospital before starting the data-collection phase.

The name of the participant was not inquired to make sure confidentiality of participant responses and access to research data was restricted to the study team only.

\subsection{Participants}

The target population were patients aged 15 - 35 who attended family medicine clinic. A total of 300 participants answered the questionnaire, the permission to fill the questionnaire was taken verbally and the purpose of the study was explained extensively before handing the questionnaire.

\subsection{Sample Size}

Patients who attended the family medicine clinic in Jordan university hospital and met the criteria for age were asked to fill up the questionnaire. The sample size was determined using the following formula:

$$
n=\frac{z^{2} \times p(1-p)}{\varepsilon^{2}}
$$

where, $n=$ sample size required for study, $z=$ standard tabulated value for $5 \%$ level of significance), $p=$ is the population proportion, and $\varepsilon=$ is the margin of error.

In this study, the total sample size of the study was calculated considering $50 \%$ prevalence at $95 \%$ confidence level with $5 \%$ allowable error. The total number of required sample size is therefore 300 .

\subsection{Statistical Analysis}

We used SPSS version 21.0 in our analysis. We used the mean ( \pm standard deviation) to describe continuous variables (e.g. age). We used count (frequency) to describe other nominal variables (e.g. gender). Comparison of categorical varia- 
bles between independent groups was done with Chi-square test. We adopted a p-value of 0.05 as a significant threshold.

\section{Results}

A total of 300 patients who attended the family medicine clinic during the period from August/2019 to October/2019 were asked to fill the questionnaire under direct supervision. The participants included in this study aged between 15 - 35 years old. $70.9 \%$ of the participants were females and $29.1 \%$ were males. Demographic characteristics of the study population are presented in Table 1.

As shown in Table 1, 26.7\% of the participants aged between 15 - 20 years old, $34.7 \%$ aged between 21 - 25 years old and 19.3\% aged between 26 - 35 years old. The majority of the participants $68.7 \%$ were not married. The participants included were from different levels of education $25.3 \%$ of them were still in high school, $60.3 \%$ had bachelor degree, $7.7 \%$ had diploma and $5.7 \%$ were postgraduates. We also included the income in our survey, the majority of the participants had income below 800 JD, 16.7\% of them had income between 800 - 1500 JD and $9.3 \%$ had income more than $1500 \mathrm{JD}$.

Table 1. The demographic characteristics of the participants.

\begin{tabular}{|c|c|c|c|}
\hline Parameter & Details & Count & Percentage \\
\hline \multirow{3}{*}{ Gender } & Female & 212 & 70.9 \\
\hline & Male & 87 & 291 \\
\hline & & & \\
\hline \multirow{5}{*}{ Age } & $15-20$ & 80 & 26.7 \\
\hline & $21-25$ & 104 & 34.7 \\
\hline & & & \\
\hline & $26-30$ & 58 & 19.3 \\
\hline & $31-35$ & 58 & 19.3 \\
\hline \multirow{3}{*}{ Marital Status } & Single & 206 & 68.7 \\
\hline & Married & 88 & 29.3 \\
\hline & Others & 5 & 1.7 \\
\hline \multirow{5}{*}{ Level of Education } & Illiterate & 2 & 0.7 \\
\hline & High school & 76 & 25.3 \\
\hline & Bachelor & 181 & 60.3 \\
\hline & Diploma & 23 & 7.7 \\
\hline & Postgraduate & 17 & 5.7 \\
\hline \multirow{4}{*}{ Income } & Less than 500 & 108 & 36.0 \\
\hline & $500-800$ & 105 & 35.0 \\
\hline & $800-1500$ & 50 & 16.7 \\
\hline & More than 1500 & 28 & 9.3 \\
\hline
\end{tabular}


Upon analyzing the data, 153 out of the 300 participants were found to have suffered or been still suffering from acne with a prevalence of $45 \%$. The mean age at which the acne started was 16.20. There was a significant correlation between age, gender and prevalence of acne, females reported acne more than males and those under the age of 25 years old reported acne more than the older age groups. Among those who answered "yes" to whether they have acne or not, 63 (41.2\%) had blackheads, 102 (66.7\%) had whiteheads and 30 (19.6\%) had cysts and nodules. There was a significant correlation between gender and prevalence of cysts and nodules, females reported having cysts and nodules more than males.

The majority of the participants 141 (92.2\%) had facial acne, 68 (44.4\%) had acne on their back and $24(15.7 \%)$ had acne on their chest. 87 (58.4\%) of affected participants reported scarring as a result of acne. Males were found to have acne on their backs more than females as shown in Table 2.

$78(51.3 \%)$ of those who had acne described it as moderate in severity, 64 (42.1\%) described it as mild and only $10(6.6 \%)$ described it as severe.

When asked about the impact of acne on quality of life, 27.5 (42\%) of affected participants believed that acne affected their self-esteem, 30 (19.6\%) of them believed that acne prevented them from attending social events, $44(28.8 \%)$ reported that acne affected their body image and 47 (30.7\%) believed that acne affected their daily life events like studying and working, as shown in Table 2. There was a significant correlation between severity of acne and impact on life, the more severe the acne, the more likely it would impact self-esteem and body image. Also, there was a significant correlation between age and impact of acne on body image, the younger the age the more they believed that having acne affected their body image.

Table 2. the prevalence of different parameters studied on acne vulgaris.

\begin{tabular}{|c|c|c|c|}
\hline Parameter & Details & Count & Percentage \\
\hline \multirow{3}{*}{ Type of Acne } & Black comedones & 63 & $41.2 \%$ \\
\hline & White comedones & 102 & $66.7 \%$ \\
\hline & Cysts and nodules & 30 & $19.6 \%$ \\
\hline \multirow{3}{*}{ Severity of Acne } & Mild & 64 & $42.1 \%$ \\
\hline & Moderate & 78 & $51.3 \%$ \\
\hline & Severe & 10 & $6.6 \%$ \\
\hline \multirow{3}{*}{ Site of Acne } & Back & 68 & $44.4 \%$ \\
\hline & Chest & 24 & $15.7 \%$ \\
\hline & Face & 141 & $92.2 \%$ \\
\hline \multirow{4}{*}{ Impact of Acne } & Self confidence & 42 & $27.5 \%$ \\
\hline & Attending social events & 30 & $19.6 \%$ \\
\hline & Impact on body image & 44 & $28.8 \%$ \\
\hline & Daily life like studying or working & 47 & $30.7 \%$ \\
\hline
\end{tabular}


More than half of the affected participants 78 (51\%) sought doctor help to treat their acne, there was a significant correlation between severity, type, scarring from acne and seeking doctor help, the more severe the acne was, the more likely patients had seen a doctor. And there was a significant correlation between gender and seeking doctor help, females were more likely to seek doctor advice. Also, those who suffered from cysts and nodules and who had acne scars were found to be more likely to seek doctor help.

As shown in Figure 1, patients depended on many sources of information to manage their acne, the majority of them 84 (54.9\%) reported the dermatologists as their source of information, the internet ranked second $41(26.8 \%)$ as a source of information, 17 (11.1\%), 16 (10.5\%) of them depended on general practitioners and on their families and friends respectively, 7 (4.6\%) of them depended on beauty centers and only $2(1.3 \%)$ depended on magazines. Patients with back acne were more likely to use magazines as a source of information and less likely to see a dermatologist. There was a significant correlation between the severity of acne, gender and the source of information, the more severe the acne was, the more likely they had gone to a dermatologist, and the less likely they had depended on family and friends for a source of information. Females went to dermatologists more than males.

As shown in Figure 2, patients tended to use different methods of treatment to manage their acne, 72 (47.1\%) used cleanser, 54 (35.3\%) used Scrubbing cream, 37 (24.2\%) used topical antibiotics, 33 (21.6\%) used oral antibiotics, 32 (20.9\%) used Roaccutane, 42 (27.5\%) used facemasks and 39 (25.5\%) used natural mixtures, only 5 (3.3\%) used laser therapy. There was a significant correlation between the severity of acne, the type of acne, the source of information and the modality of treatment used, the more severe the acne was, the more likely scrubbing creams, oral antibiotics, and Roaccutane were used. Those who had whiteheads were more likely to be treated with topical antibiotics in contrast to those who had cysts and nodules that were more likely to be treated with oral antibiotics. Patients who answered "yes" to whether they had seen a doctor were more likely to use scrubbing creams, topical antibiotics, oral antibiotics, and Roaccutane. Patients who used the internet as their source of information were more likely to use a cleanser and less likely to use Roaccutane. Patients who attended beauty centers were more likely to use facemasks. Patients who depended on family and friends as their source of information were less likely to use oral antibiotics. Acne scars were more likely to be treated with scrubbing creams. Also, there was a significant correlation between gender and modality of treatment; females were more likely to use facemasks, natural mixtures and Roaccutane. Lastly, Patients who sought general practitioners as their source of information were less likely to use cleansers or Roaccutane, in contrast, those who sought dermatologists as their source of information were more likely to use scrubbing creams, oral antibiotics, and Roaccutane.

When asked about how much money they spent on acne treatment per 
month, 66 (44\%) of them spent less than $20 \mathrm{JD}$ on treatment, $43(28.7 \%)$ spent about 20 - 50 JD, 5 (3.3\%) spent about 50 - 100 JD, 4 (2.7\%) spent more than 100 JD and $32(21.3 \%)$ of the affected participants spent nothing on treatment. There was a significant correlation between gender and the amount of money spent on acne treatment, females spent more money than males to treat acne.

You can refer to Table 3 to see the summary of the significant p-value.

\section{Discussion}

Acne is a common dermatological issue among Jordanian adolescents and young adults aged between 15 and 35 years old, in our study the prevalence was $45 \%$ which is similar to other studies held in Syria34\%, turkey $40.1 \%$ and Saudi Arabia $56.2 \%$ [6].

The findings of high prevalence of mild to moderate acne vulgaris were consistent with many studies. A similar pattern was obtained in comparative studies done in Queens Medical Centre, Nottingham in UK where (50\%) were rated by the researcher as having no acne, (22\%) as having very mild acne, (17\%) as having mild acne and $34(11 \%)$ as having moderate to severe acne [7], another study conducted in Nigeria showed only one student had severe acne vulgaris (GAGS, 31-38), 10\% moderate (GAGS, 19-30), and 89.4\% mild (GAGS, 1-18) [8].

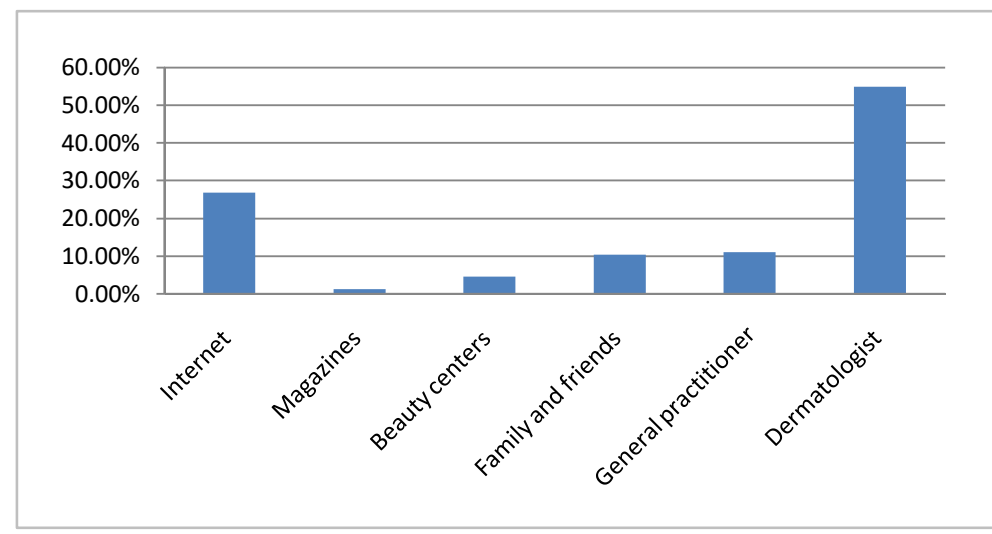

Figure 1. Sources of information.

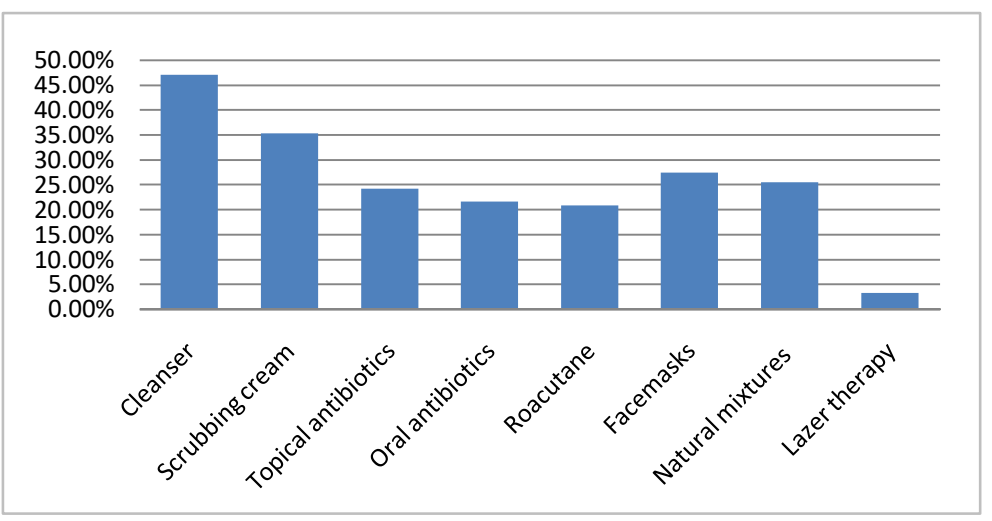

Figure 2. Modalities of treatment. 
Table 3. Summary of the significant p-values in the article.

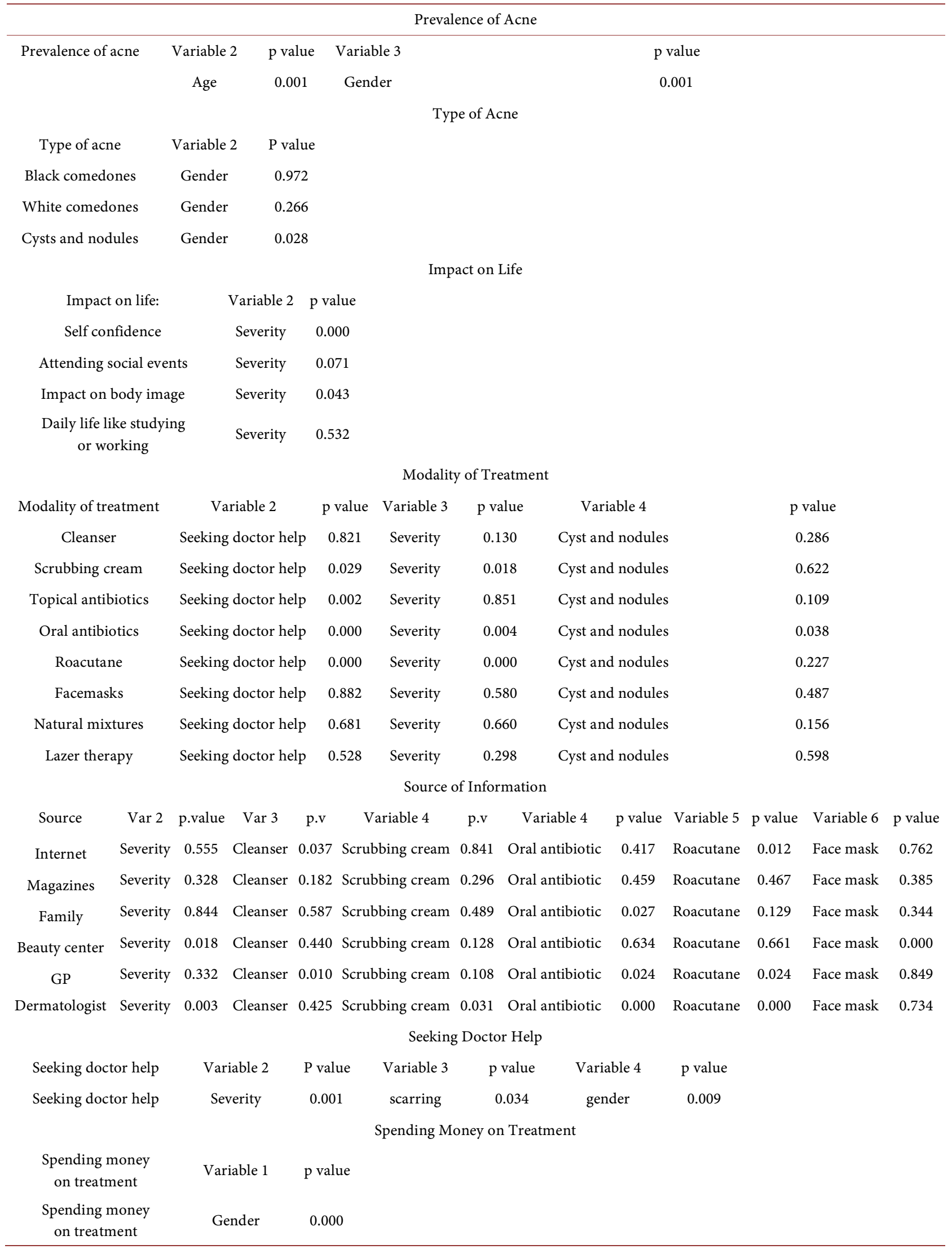


The mean age of acne vulgaris among participants was 16 year old which is similar to findings documented in previous studies. For example, a Syrian study showed that the mean age of students with acne was significantly younger in males $(18.13 \pm 0.59)$ than in females $(19.04 \pm 1.10)$ [6], also among a total of 10 521 participants across 7 European countries the prevalence of acne was highest in the 15- to 17-year-old age class and decreased with increasing age [9]. Another huge study compared the epidemiology of acne vulgaris among Caucasian, Asian, Continental Indian and African American women showed that acne peaked between the ages of 16 and 20 years in all racial groups excluding Hispanic women, in whom acne peaked slightly later [7].

According to our study females were more likely to have acne than males which is inconsistent with other studies held in Syria and UK [6] [10] where males were more likely to get acne, on the other hand, females were more likely to get acne in a Saudi Arabian study [11]. A recent Australian study, however, reported negligible sex differences in prevalence between the ages of 13 and 15 years, with rates of $77 \%$ for boys and $79 \%$ for girls. In the $16 \pm 18$-year age group, however, males in that study were more likely to have acne and males had more severe acne overall [12]. This discrepancy could be due to cultural underestimations of acne status and body image among males especially teenagers. Our results showed no statistically significant association between gender and severity.

Regarding psychological and social effects of acne vulgaris, our results showed a significant association between severity of acne and low self-esteem and impact on body image but severity did not affect attending social events or daily activity. A similar study to ours was held in Greece about the impact of acne vulgaris on quality of life and psycholigical health in young adolescents; it was found that the impact of acne on quality of life is associated with the severity of the acne ( $\mathrm{p}<$ 0.0001). Patients with moderate/severe acne experience greater psychosocial and emotional impairment $(\mathrm{p}<0.0001)$. Body image is modified proportionally to the severity of acne $(\mathrm{p}<0.0001)$. It also concluded that symptoms and treatment of acne are factors that influence quality of life [13]. Another study was conducted at The Oxford Medical College, Hospital \& Research Center (Bangalore, India) showed that acne vulgaris is a disease that can adversely affect an individual's quality of life and self-esteem. It emphasized the importance of screening for psychosocial problems in those who present for management of acne [14]. A literature review, in which 13 articles that examined the effect of acne vulgaris on patients' self-esteem were reviewed, concluded that acne vulgaris has a substantial negative impact on patients' self-esteem. And more severe acne vulgaris and acne in women tended to have the greatest impact across cultures. It also concluded that effective treatment improves patients' self-esteem [15].

Not surprisingly, acne severity and presence of scarring influenced the tendency to seek medical care; those who perceived their acne to be more frequent and severe were more inclined to seek a physician's treatment advice. We also identified gender differences in help seeking behavior; females were more likely 
to seek doctor advice. This finding has been consistently reproduced by other studies [5] [10].

Despite the high psychological morbidities caused by acne, adults did not view acne as a medical condition and underused treatments, Furthermore, they did not seek medical attention, with help-seeking rates found to be $<30 \%$ in most studies like a Self-reported survey with participants drawn from 120 adolescents aged 13 - 18 in a London tertiary pediatric outpatient department and 482 adolescents from two London schools, aged 11 - 18 reported that they had never sought help from health professionals for management of acne [16]. Also an American study held in New Jersey showed that only $17 \%$ of participants sought medical help for acne vulgaris [5].

Our study provided further evidence of the gap in medical care in adults with acne with only $51 \%$ of them had sought medical help. The reason behind that maybe because most of our participants were visitors of JUH family center where they are insured and rarely we do find visitors who are not insured.

The most frequently reported method of managing acne was non-prescribed products mainly cleansers, scrubbing and face masks as showed by other studies. The use of over-the-counter cosmetic remedies in our study, as in that by Yahya, was widespread. Our findings indicating that females used local acne treatment more often than males concur with the findings of Nijsten et al. [4] [17].

In this study the most frequently used non-medical source to gain knowledge about acne was the internet, however another study showed that non-medical sources included magazines (44\%), television (44\%), parents (31\%), and friends $(28 \%)$ were most frequently used [18].

\section{Limitations}

Our study depended on self-reported grading of acne severity as mild, moderate or severe which may vary according to the age, gender, psychological effect and level of education. Also, the psychological morbidity of acne vulgaris could be underestimated because we did not include a validated score for self-esteem and quality of life in the questionnaire.

\section{Conclusion}

Our article demonstrates that acne is a prevalent distressing problem among adolescents; it affects patients' quality of life. Different modalities of treatment and sources of information were sought by patients. As family doctors, we emphasize the need for accessible, accurate, community-based education on the modalities of treatment of acne, and the importance of prompt medical attention toward its effect on quality of life.

\section{Conflicts of Interest}

The authors declare no conflicts of interest regarding the publication of this paper. 


\section{References}

[1] Purdy, S. and de Berker, D. (2011) Acne Vulgaris. BMJ Clinical Evidence, 2011, 1714 .

[2] Cunliffe, W.J. and Gould, D.J. (1979) Prevalence of Facial Acne Vulgaris in Late Adolescence and in Adults. British Medical Journal, 1, 1109-1110.

https://doi.org/10.1136/bmj.1.6171.1109

[3] Szepietowski, J., et al. (2018) Acne across Europe: An Online Survey on Perceptions and Management of Acne. Journal of the European Academy of Dermatology and Venereology, 32, 463-466. https://doi.org/10.1111/jdv.14719

[4] Hun Suh, D., et al. (2009) Treatment-Seeking Behaviors and Related Epidemiological Features in Korean Acne Patients. Journal of Korean Medical Science, 23, 969-974. https://doi.org/10.3346/jkms.2008.23.6.969

[5] Corey, K.C., et al. (2013) Self-Reported Help-Seeking Behaviors and Treatment Choices of Adolescents Regarding Acne. Pediatric Dermatology, 30, 36-41. https://doi.org/10.1111/j.1525-1470.2012.01807.x

[6] Al-Kubaisy, W., et al. (2014) Sociodemographic Characteristics of Acne among University Students in Damascus, Syria. Epidemiology Research International, 2014, 4. https://doi.org/10.1155/2014/974019

[7] Perkins, A.C., et al. (2011) Comparison of the Epidemiology of Acne Vulgaris among Caucasian, Asian, Continental Indian and African American Women. Journal of the European Academy of Dermatology and Venereology, 25, 1054-1060. https://doi.org/10.1111/j.1468-3083.2010.03919.x

[8] Akinboro, A.O., et al. (2018) The Impact of Acne and Facial Post-Inflammatory Hyperpigmentation on Quality of Life and Self-Esteem of Newly Admitted Nigerian Undergraduates. Clinical, Cosmetic and Investigational Dermatology, 11, 245-252. https://doi.org/10.2147/CCID.S158129

[9] Wolkenstein, P., Szepietowski, J.C., Tennstedt, D., Veraldi, S. and Delarue, A. (2017) Acne Prevalence and Associations with Lifestyle: A Cross-Sectional Online Survey of Adolescents/Young Adults in 7 European Countries. https://doi.org/10.1111/jdv.14475

[10] Ac, S., Glazebrook, C. and Williams, H.C. (2001) Acne Prevalence, Knowledge about Acne and Psychological Morbidity in Mid-Adolescence: A Community-Based Study. The British Journal of Dermatology, 145, 274-279. https://doi.org/10.1046/j.1365-2133.2001.04346.x

[11] Darwish, M.A. and Al-Rubaya, A.A. (2013) Knowledge, Beliefs, and Psychosocial Effect of Acne Vulgaris among Saudi Acne Patients. ISRN Dermatology, 2013, 6. https://doi.org/10.1155/2013/929340

[12] Kilkenny, et al. (1998) The Prevalence of Common Skin Conditions in Australian School Students: 3. Acne Vulgaris. British Journal of Dermatology, 139, 840-845. https://doi.org/10.1046/j.1365-2133.1998.02510.x

[13] Tasoula, E., et al. (2012) The Impact of Acne Vulgaris on Quality of Life and Psychic Health in Young Adolescents in Greece. Results of a Population Survey. Anais Brasileiros de Dermatologia, 87, 862-869. https://doi.org/10.1590/S0365-05962012000600007

[14] Hosthota, A. and Basavaraja, V. (2016) Impact of Acne Vulgaris on Quality of Life and Self-Esteem. Cutis, 98, 121-124.

[15] Gallitano, S.M. and Berson, D.S. (2017) How Acne Bumps Cause the Blues: The Influence of Acne Vulgaris on Self-Esteem. International Journal of Women's Der- 
matology, 4, 12-17. https://doi.org/10.1016/j.ijwd.2017.10.004

[16] Desai, K.P., et al. (2017) Help-Seeking Behaviours, Opportunistic Treatment and Psychological Implications of Adolescent Acne: Cross-Sectional Studies in Schools and Hospital Outpatient Departments in the UK. BMJ Open, 7, e016964. https://doi.org/10.1136/bmjopen-2017-016964

[17] Nijsten, T. and Lambert, J. (2007) Acne Is Prevalent But Use of Its Treatments Is Infrequent among Adolescents from the General Population. https://doi.org/10.1111/j.1468-3083.2006.01829.x

[18] Tan, J.K.L., Vasey, K. and Fung, K.Y. (2001) Beliefs and Perceptions of Patients with Acne. Journal of the American Academy of Dermatology, 44, 439-445.

https://doi.org/10.1067/mjd.2001.111340 


\section{Appendix}

This is a cross sectional study that aims to detect the prevalence of acne and its treatment modalities.

Kindly, answer the following questions completely.

- Age?

o $15-20$

o $21-25$

o $26-30$

o $31-35$

- Gender?

o Female

o Male

- Educational level?

o Illiterate

o Primary school

o Bachelor

o Diploma

o Higher studies

- Marital status?

o Single

o Married

o Others

- Monthly income?

$0<500$

○ $500-800$

o $800-1500$

o $>1500$

- Have you had acne in the past?

o No

o Yes, at any age ...

- Have you had current acne?

o No

o Yes, when did it started ...

If you have answered yes to any of the previous 2 questions, kindly answer the rest of the questionnaire:

- What type of acne was it?

o Black heads

0 White heads

o Cysts and pustules

- Did you suffer from any acne scars?

o No

o Yes

- You do describe your acne as?

o Mild 
o Moderate

o Severe

- Where is/was the distribution of your acne?

o Face

o Chest

o Back

o Others, mention ...

- Choose one or more of the following effects acne had on you?

o Your self confidence

o Attending social events

o Impact on body image

o Daily life like studying or working

- Did you seek any medical advice regarding acne before?

o No

o Yes

- What resources do you depend on to help treating your acne "you can choose more than one"?

o Websites

o Magazines

o Beauty centers

o Family and friends

o General practitioner

o Dermatologist

- Which products have you used for treating your acne "you can choose more than one"?

o Cleansers

o Peeling products

o Topical antibiotics

o Oral antibiotics

o Roaccutane

o Facial masks

o Herbals and home remedies

o Laser treatment

- How much do you spend monthly on your skin, on average?

$0<20$ JDs

○ $20-50$

○ $50-100$

$0>100$

o Nothing

Thanks for participating. 